\title{
Long Descending Lymphatic Pathway from the Pancreaticoduodenal Region to the Para-aortic Nodes: Its Laterality and Topographical Relationship with the Celiac Plexus
}

\author{
By
Ichiro HIRAI ${ }^{1)}$, Gen MURAKAMI ${ }^{2)}$, Wataru KIMURA ${ }^{1)}$, Takashi NARA ${ }^{\text {() }}$ and Yukio DODO ${ }^{3)}$

\footnotetext{
${ }^{1)}$ Department of Surgery, Yamagata University School of Medicine,

${ }^{2)}$ Department of Anatomy, Sapporo Medical University School of Medicine,

${ }^{3)}$ Department of Anatomy, Tohoku University Graduate School of Medicine
}

- Received for Publication, December 5, 2000 -

\begin{abstract}
Key Words: Paraaortic lymph nodes, Hepatoduodenal ligament, Pancreaticoduodenal region, Thoracic duct, Lymphadenectomy

Summary: In 6 of 15 postmortem-treated cadaveric specimens, we found macroscopically thick lymphatic collecting vessels that originated from not only the nodes along the common hepatic artery (No. 8 nodes) but also from the pancreaticoduodenal region, and which drained directly into the para-aortic nodes immediately below the left renal vein (No. 16b1-inter or -latero nodes). The collecting vessels, if they originated from the ventral (dorsal) visceral side, passed to the left (right) of the superior mesenteric and celiac arteries. Moreover, the right-side vessels (5 specimens) were classified into superficial and deep courses to the celiac plexus, whereas they were superficial in the left side $(2$ specimens). One of the deep (right) courses continued to the thoracic duct without any intercalated nodes. In addition, another deep route drained into the para-aortic node immediately above the left renal vein (No. 16a2-inter node). We consider that these collecting vessels form "direct descending pathways" from the relatively peripheral lymphatics in the upper abdomen toward the thoracic duct origin. The pathway seems to be a collateral, or even major drainage route, and it appears responsible for skipped metastasis of primary cancer. Since the classical, limited entity of the intestinal lymph trunk does not coincide with our pathway, it should be reconsidered. The proposed entity of the direct, long descending pathway will influence the selection and modification of lymphadenectomy methods in cancer surgery in the pancreaticoduodenal region.
\end{abstract}

In primary cancer of the bile duct, the hilar portion of the liver or the pancreaticoduodenal region, early metastasis is frequently found in the lymph nodes that are located in the dorsal aspect of the hepatoduodenal ligament and pancreatic head (Kayahara et al., 1992; Nakao et al., 1995), i.e., Nos. $12 \mathrm{p}, 8 \mathrm{p}$ and 13 nodes according to the general classification of lymph nodes in Japan (Japanese Research Society for Gastric Cancer, 1995; see Fig. 1). Previous macroscopic studies (Inoue, 1936; Mukao, 1954; Hiraki, 1958b; Kida, 1958; Tanigawa, 1963; Durovicova and Munka, 1973), which were based on dye injection into the embryonic intestinal nodes, demonstrated that the drainage route from those dorsal node groups forms a tributary (radicle or root) of the intestinal lymph trunk. Therefore, it is likely that one or more, thick, efferent pathway(s) of the dorsal nodes directly reach the abdominal para-aortic nodes, i.e., No. 16 nodes according to Japanese Research Society for Gastric Cancer (1995). Moreover, previous studies using embryos exhibited that the efferent vessels not only empty into No. 16 nodes but also, sometimes, directly connect the thoracic duct and/or join the lumbar lymph trunk (Jossiifow, 1906, 1930: Hiraki, 1958a, 1958c; Mukao, 1954; Tanigawa, 1963). Those studies using embryos are excellent but the results might not always correspond to the topographical anatomy in adults. The celiac plexus and fascial arrangement are especially difficult to identify in embryos.

In contrast, according to recent macroscopic

Corresponding to: Ichiro Hirai, Department of Surgery, Yamagata University School of Medicine, 2-2-2 lida-nishi. Yamagata 990-9585. Japan. Grant sponsor: Grant-in-aid for Scientific Research. Ministry of Education. Science, and Culture; Grant number: $1267-0024$ 


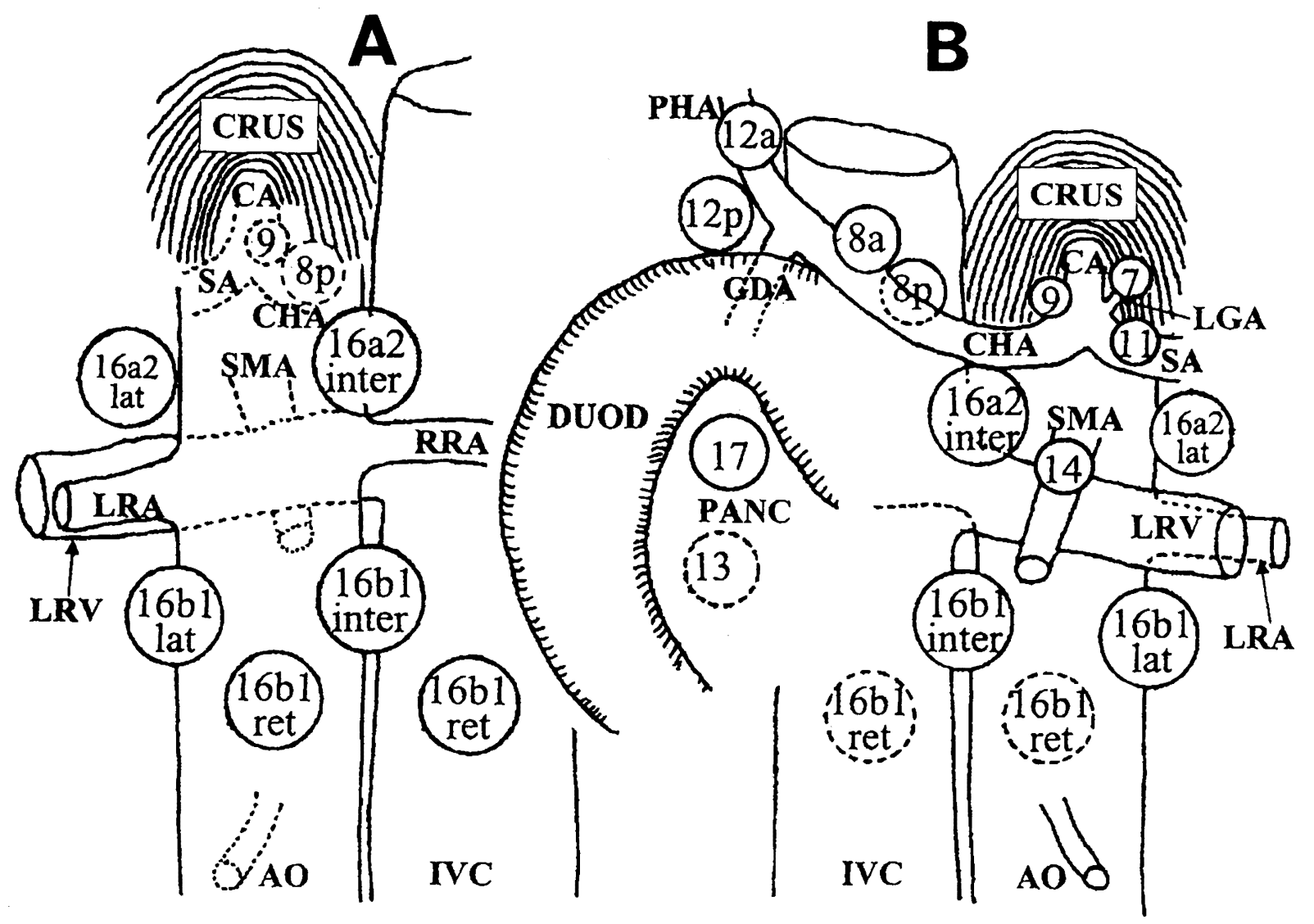

Fig. 1. General classification of the abdominal lymph nodes in Japan (Modification of Japanese Research Society for Gastric Cancer, 1995).

A. Dorsal view; B, Ventral view. In subgroups of the paraaortic nodes, 5 limited subgroups that are located immediately above and below the left renal vein (above, No. 16a2-inter node and No. 16a2-latero node; below, No. 16b1-inter node, No. 16b1-latero node and No. 16b1-retro node are depicted). No. 16 nodes, except for No. 16b1-retro node, are likely to distribute or extend from the ventral side to the dorsal side. No. 13 (No. 17) node is located at the dorsal (ventral) surface of the pancreatic head. Nos. 7, 8, 9, 11, 12, 14 nodes are located along an artery or vein. The subgroups of No. 14 node are omitted. AO, aorta; CA, celiac artery (celiac trunk); CHA (PHA), common (proper) hepatic artery; CRUS, diaphragmatic crus; DUOD, duodenum; IVC, inferior vena cava; LGA, left gastric artery; LRA (LRV), left renal artery (vein), PANC, pancreas; SA, splenic artery; SMA, superior mesenteric artery.

studies using adult cadavers (Sato and Sato, 1986; Sato et al., 1988b; Deki and Sato, 1988; Ito et al., 1991: Deki et al., 1991; Ito and Mishima, 1994; Sato, 1997), drainage routes from Nos. $12 p, 8 p$ and 13 nodes seem to comprise of a network of lymphatic vessels around the celiac and superior mesenteric arteries rather than a pathway formation to communicate directly with No. 16 nodes. That lymphatic network contains several intercalated nodes such as Nos. 9 and 14 nodes. Actually, Ito et al. (1991) and Ito and Mishima (1994) proposed entities of the pathway, i.e., the cholecysto-retropancreatic pathway in the right side and the cholecysto-celiac pathway in the left side. However, we speculated that their entity of the pathway is not based on the direct, thick and long collecting vessels but on the network composed of numerous lymphatic vessels and nodes. If their pathway is present, why did they not present photographs? Consequently, in adults, it is still unclear whether the pathway from the dorsal nodes to No. 16 nodes is present or not in the dorsal aspect of the pancreaticoduodenal region (i.e., the right of the celiac and superior mesenteric arteries after retroduodenal mobilization of the fusion fascia: see below and Materials and Methods).

Although a small, limited area was shown at and around the abdominal viscera, Hidden and Hureau (1978), Sarrazin et al. (1980) and Pissas (1984) suggested a lymphatic pathway even in adults by using an injection method for fresh cadavers. Nagai (1987) and Uesaka et al. (1996) also demonstrated a pathway in the dorsal (right) side of the pancreaticoduodenal region using carbon suspension injection during surgery. Those studies are excellent for their demonstrations of specific drainage routes, but we consider that their results are usually 
overestimated rather than the physiological condition. Takayama et al. (2000) reported variations in physiological lymphatic flow originating from the stomach by using oral administration of excess lipid in combination with the regional injection of carbon particles during surgery. Their results indicated that the carbon may be able to pass through the node even under the physiological condition. Moreover, injection studies generally seem to support the network theory (see above) rather than the pathway since numerous branches as well as the "pathway" were also made clear by injection.

Nervertheless, injection studies stressed the existence of lymphatic pathways. Recent clinical reports about skipped lymph node metastasis from primary cancers of the upper abdominal organs also seem to support the existence of collateral route or pathway (Abstract of the 24th Research Meeting of the Lymph Node Metastasis of Cancer, 1996). Moreover, the dorsal node groups are usually developed well and located near the thoracic duct origin, i.e., No. 16 nodes. The close topographical relationship between the dorsal nodes and paraaortic region is similar to that in the posterior mediastinum in which multiple (not a single) bronchmediastinal lymph trunks are evident and join the thoracic duct (Murakami et al., 1990; Fujii et al., 1994). Consequently, we believed that a lymphatic pathway is present in the region and that we can demonstrate and take a photograph of the pathway as one or $2-3$ trunks.

Recently, there is a worldwide trend toward minimumly-invasive surgery, and some surgeons believe that Kocker's maneuver (separation and mobilization of the fusion fascia behind the duodenum: see also Materials and Methods) can allow them to operate on the entire or most of the lymphatic drainage routes from the hepatoduodenal ligament and/or the pancreaticoduodenal region to the para-aortic region (Pitt, 1995; Henne-Brus et al., 1998; Yeo et al., 1999). Moreover, some anatomists and surgeons believe that the drainage routes from Nos. $8 \mathrm{p}$ or 13 nodes are located superficial to the celiac plexus and ganglion (Sato, 1997; Nagai et al., 1991). Thus, such minimumly-invasive surgery tends to include the preservation of the plexus. Conversely, lymph node dissection tends to be conducted within the superficial lymphatics under the relatively limited operative field obtained using Kocker's maneuver (Kondo et al., 1998). The operative field which is able to be approached by Kocker's maneuver includes the dorsal aspect of the pancreaticoduodenal region, the right side of the origin of the celiac and superior mesenteric arteries and most of the para-aortic region, but it excludes the dorsal and left sides of the aorta.
Nevertheless, neither a suggestive lymphatic pathway (see above) nor a kind of topographical shift or difference in density of the suggestive lymphatic network have not been suggested by previous studies. Moreover, the anatomy of the collateral or skipped lymphatic route has not been investigated in the region, raising the question of how we can discuss the reliability and limitations of minimumlyinvasive surgery without critical anatomical information.

Overall, the aim of this study is to demonstrate the lymphatic collecting vessels, especially the pathway from the Nos. $8 p$ and/or 13 nodes to the No. 16 nodes and to reveal the topographical anatomy of the pathway. The discussion will concentrate on whether the pathway is able to be approached and operated on within the relatively-limited operative field obtained by Kocker's maneuver. This is a preliminary report of dissections using 6 cadaveric specimens.

\section{Materials and Methods}

Fifteen specimens of the entire abdominal viscera were obtained from postmortem-treated Japanese cadavers (10 males and 5 females), aged 65-78 years old when they died and which had been donated for medical education and research to Sapporo Medical University (12 specimens) or Tohoku University School of Medicine (3 specimens). In 7 of the 15 , to easily discriminate the lymphatic vessels from the veins on the visceral surface, a water-soluble resin (absolute solution of Latex 601-A, DuPont Dow Elastomers, Tokyo) had been injected into the entire portal vein system from the ileocolic vein before the postmortem treatment with arterial perfusion of $10 \%$ formalin solution (Murakami et al., 1999).

Dissection and demonstration of lymphatics were performed with the naked eye with aid of a binocular microscope according to Deki and Sato (1988). After we performed Kocker's maneuver or retropancraticoduodenal mobilization (a manual separation of the retroperitoneal fusion fascia behind the pancreatic head and duodenum), we selected 8-10 good specimens for observation of lymphatics according to whether lymph vessels (not lymph nodes) were developed well in the dorsal aspect of the pancreaticoduodenal region. However, we also eliminated specimens in which the paraaortic lymph nodes were too highly developed to separate them from each other. Second, from the dorsal side of the abdominal viscera, the thoracic duct and its major origin were dissected including all lymph nodes around the abdominal aorta and 
inferior vena cava. Third, to reveal lymphatic communications, we dissected in detail the dorsal or right aspect of the pancreatic head, the duodenum, the celiac and superior mesenteric arteries. From the right side, the celiac plexus and ganglia were removed after depiction of the superficial lymphatics. Finally, we examined whether thick collecting vessels were present on the left or ventral side of those two arteries. The liver, stomach and most of the intestine were gradually removed to obtain a good visual field depending on the progress of dissection. During dissection, we paid much attention to maintain continuation of the efferent lymphatics from Nos. $8 p$ and 13 nodes in the pancreaticoduodenal region to No. 16 nodes or the thoracic duct itself. Observations were depicted by photographs step-by-step.

To make sure that there were no intercalated nodes along the collecting lymph trunks, if they were present, clearing using methyl salicylate was performed after dehydration with absolute ethanol (Murakami et al., 1994). We examined 2 vessels that were found on the right side in 2 specimens (from No. 8p node via the superficial side of the celiac plexus to No. 16b1-inter node) and one on the left side (near the splenic vein origin to No. 16b1-inter node). Before employing the clearing method, the lymphatic valves were examined in water under a binocular microscope (Fujii et al., 1994). However, the results were omitted in the following text since neither intercalated nodes nor clearly-identifiable valves were evident in any case. Moreover, after the clearing, we histologically examined (routine paraffin embedding and H.E. staining) the connection between the macroscopically-identified lymphatic vessels and the marginal sinus of the node to confirm our observations (data, not shown).

\section{Results}

In 6 of the 15 specimens examined, thick collecting vessels were evident on the right side of the celiac and superior mesenteric arteries after Kocker's maneuver (Figs. 2-4). We termed this the "right long descending pathway." In all cases of the right long descending pathway, the drainage territory included the dorsal aspect of the pancreaticoduodenal region and No. $8 \mathrm{p}$ node (dorsal group, the nodes along the common hepatic artery). The right long descending pathway was classified into superficial and deep courses according to whether the collecting vessel ran superficially (in 5 of the 6 specimens) or deeply (in 3 of the 6) to the celiac plexus and ganglion (Table 1). Both courses
Table 1. Courses of the right descending lymphatic routes originating from No. $8 p$ node and/or dorsal aspect of the pancreaticoduodenal region and draining into No. 16b1-inter and/or No. 16b1-latero nodes

\begin{tabular}{|c|c|c|}
\hline Superficial $^{\text {a) }}$ course only & 3 & $\begin{array}{l}\text { (into } 16 \mathrm{~b} 1 \text {-int, } 2 \text { specimens; } 16 \mathrm{~b} 1- \\
\text { lat, } 1 \text { specimen }^{\mathrm{b})} \text { ) }\end{array}$ \\
\hline Deep $p^{\text {a) }}$ course only & 1 & $\begin{array}{l}\text { (direct to TD + communication } \\
\text { with } 16 \mathrm{~b} 1 \text {-int })^{\mathrm{c})}\end{array}$ \\
\hline Both & 2 & $\begin{array}{l}\text { (into 16b1-int only, } 1 \text {; both into } \\
16 \mathrm{~b} 1 \text {-int and lat, } 1^{\text {d) }} \text { ) }\end{array}$ \\
\hline
\end{tabular}

a: Superficial or deep to the celiac plexus and ganglion.

b and d:all 5 superficial collecting vessels corresponded to the "right long descending pathway" with no intercalated nodes. $d$, the deep course was not a pathway but a node chain. Specimen b, see Fig. 2; c, see Fig. 4; d, see Fig. 3. int, inter: lat, latero; TD, thoracic duct.

coexisted in 2 specimens.

The superficial course had no intercalated nodes along its course from the No. $8 \mathrm{p}$ and/or No. 13 (nodes on the dorsal surface of the pancreatic head) to one of the No. 16 nodes (Figs. 2 and 3). The superficial collecting vessel was thicker than the deep one when both were present. Some tributaries of the superficial vessels accompanied the communicating nerve branch between the celiac and renal plexuses, whereas in 2 of the 3 specimens with the deep course, the route was composed of a lymph node chain embedded in tight connective tissue between the celiac plexus and diaphragmatic crus (Fig. 3). Thus, those 2 cases were not regarded as the "long pathway." However, most of the intercalated nodes were difficult to discriminate from the usual No. $16 \mathrm{a} 2$ nodes due to the close relations with the aorta, although, in general, No $16 \mathrm{a} 2$ is believed to be located superficial to the plexus. The deep descending lymphatics seemed to receive lymph from a wider drainage territory than the superficial one, i.e., a territory including not only Nos. 8 p and 13, but also possibly, No. 7 node along the left gastric artery and vein.

On the right side, consequently, the long descending "pathway" (i.e., the thick collecting vessel without intercalated nodes) drained into the 16b1latero node and/or No. 16b1-inter node, regardless of whether it showed the superficial or deep course to the celiac plexus. However, notably, in one specimen (Fig. 4), the right deep descending route was comprised of a bundle of collecting vessels without any intercalated nodes, as demonstrated by the clearing method as well as macroscopically and, moreover, it connected with the thoracic duct.

On the left side of the celiac and superior mesenteric arteries, in 2 specimens, a single, thick collecting vessel was evident (Fig. 3C). The vessel was located superficial to the left renal vein and the ce- 

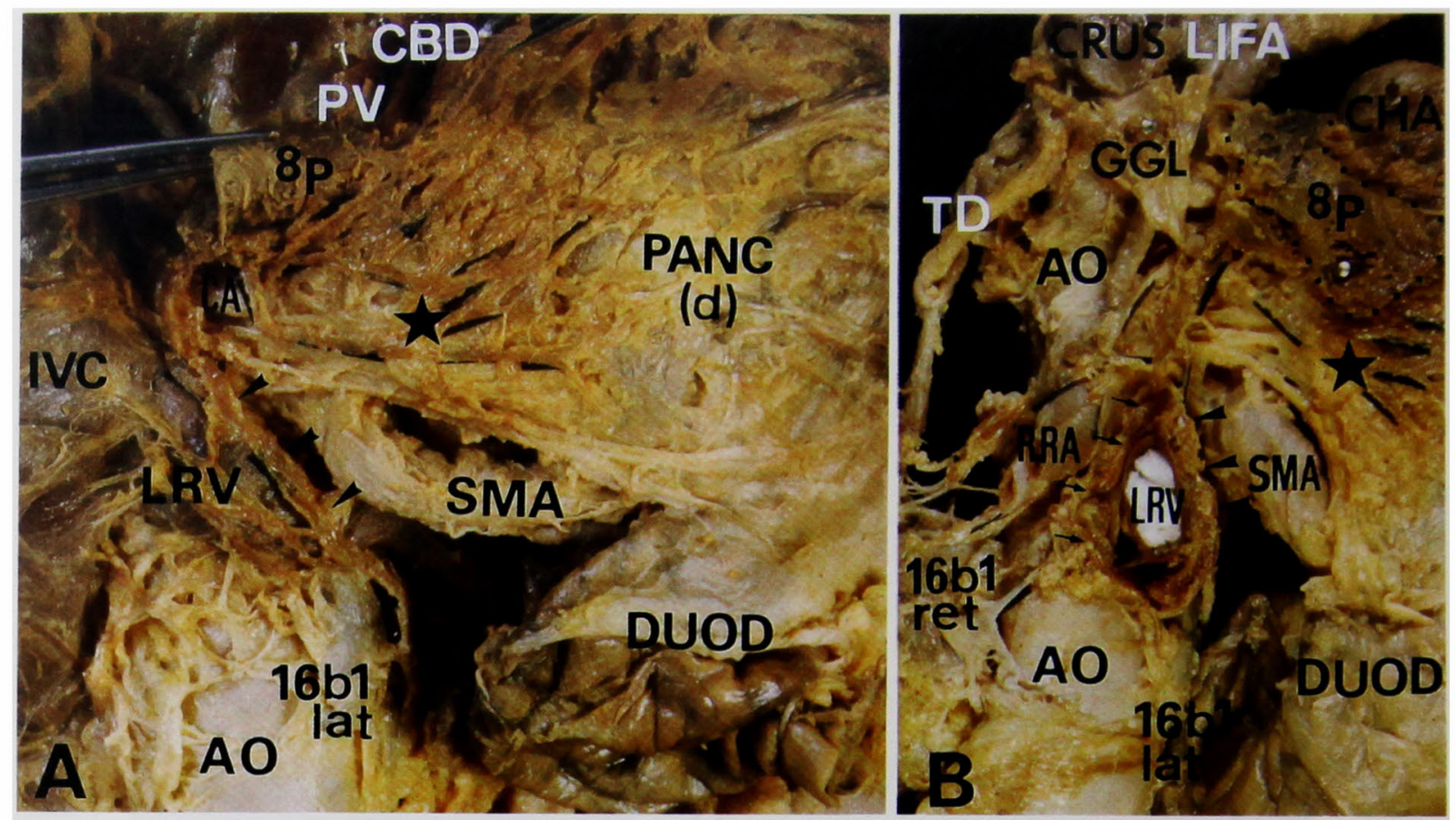

Fig. 2. Superficial collecting trunks draining into No. 16b1-latero node.

A. Numerous lymph collecting vessels originate from No. $8 p$ node $(8 p)$ as well as the posterior surface of the pancreatic head (PANC, $d$ in parentheses). Those vessels join together and form a thick trunk (arrowheads). The trunk is located superficial to most of the celiac plexus. The trunk crosses the left renal vein (LRV) and empties into No. 16b1-latero node (16b1 lat). The location of the star corresponds to that in Fig. B. AO, aorta; CA, celiac artery; CBD, common bile duct; DUOD, third portion of the duodenum; IVC. inferior vena cava; PV, portal vein; SMA, superior mesenteric artery.

B. Another deep collateral trunk (arrows) was found dorsal to the LRV (white paper was inserted into the opening of the vei.. ) after removal of the IVC. The superficial trunk shown in Fig. A is indicated by arrowheads. The collateral trunk empties into No. 16b1-lat as well as No. 16b1-retro node (16b1 ret). The celiac ganglion (GGL) was cut and partly reflected upward to show the deep lymphatics. The left inferior phrenic artery (LIFA) is evident due to its direct origin from the AO. The thoracic duct (TD) originates from 16b1-ret. No trunk was found in the left side of the SMA and CA in this specimen. CHA, common hepatic artery; CRUS, diaphragmatic crus; RRA, right renal artery (cut).

liac and superior mesenteric plexuses. In contrast to the right-side lymphatics, the left route received lymphatic vessels from the ventral aspect of the pancreaticoduodenal region including No. 8 a node (ventral group of the nodes along the common hepatic artery). These collecting lymphatic vessels along the splenic vein and left gastric artery also merged with the left pathway. Moreover, a thick collecting vessel along the superior mesenteric vein also emptied into the left pathway near the terminals of the splenic vein in the case shown in Fig. 3C, although we did not pay much attention to maintain intact the lymphatic routes along the superior mesenteric artery and/or vein. In both specimens, they emptied into No. 16b1-inter node after joining the right pathway. No intercalated nodes were present from the level of the celiac artery to No. 16b1-inter node.

The subgroups of No. 16 nodes communicated with each other. In particular, No. 16b1-inter node and No. 16b1-latero node were connected by thick vessels. Those two subgroups received not only the long descending pathway but the lumbar lymph trunks. Moreover, the latter communicated with the pathway by fine vessels, or even joined together, before terminating at No. 16 nodes.

In the other 9 specimens without the long descending pathway, thick collecting vessles were not found to the right of the celiac and superior mesenteric arteries except for around No. $16 \mathrm{a} 2$ inter node. However, thin or thick, but short afferent lymphatic vessels were seen originating from the celiac plexus itself or the deep side of the plexus (i.e., the tight connective tissue between the plexus and diaphragmatic crus) and draining into No. 16a2 inter node. (Fig. 5). The afferent vessels of No. 16a2-inter node seemed to penetrate or run deep to the celiac plexus. However, we could not dissect them to the peripheral side. Histology revealed that numerous, thin vessels not containing red blood cells were located under the plexus (not shown).

Those results are summarized in Fig. 6. In spite 

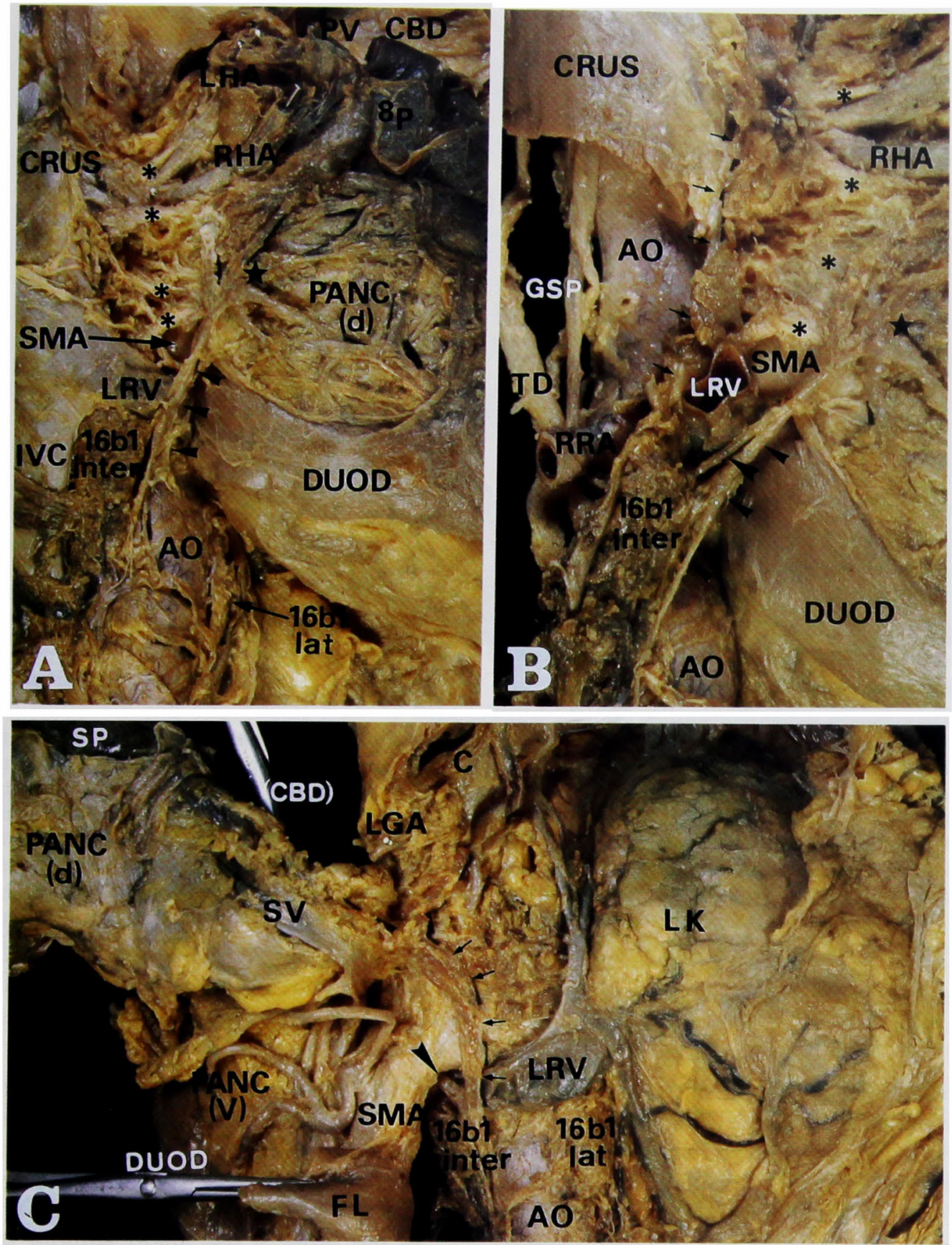

Fig. 3. Deep and superficial lymphatics draining into No. 16bi-inter node in the right side in combination with the well-developed lymph trunk in the left side.

A. Superficial lymphatics from No. $8 p$ node $(8 p)$ and the posterior surface of the pancreatic head (PANC, $d$ in parentheses) is evident as shown in Fig. 2. However, the superficial collecting trunk (arrowheads) empties into No. 16b1-inter node (16b1 inter) as well as No. 16b1-latero node (16b1 lat). The locations of the star, asterisks and arrowheads correspond to those in B. Asterisks, celiac plexus; LHA, left hepatic artery from the celiac trunk; RHA, right hepatic artery from the celiac trunk. Other abbreviations are same as in Fig. 2.

B. A deep lymph node chain (arrows) is observed between the celiac plexus and CRUS after removal of the IVC. The deep chain crosses dorsal to the LRV and drains into No. 16b1-inter. Another superficial trunk (large arrowhead) appears behind the trunk shown in A. The celiac plexus (asterisks) was partly removed. The thoracic duct (TD) originates from 16b1-inter. A collateral of the TD accompanies the greater splanchnic nerve (GSP). RRA, right renal artery (cut).

C. A thick lymph trunk is found in the left side of the SMA and celiac trunk. This trunk is formed by joining of collecting vessels along the splenic vein (SV) and left gastric artery (LGA). The left trunk empties into No. 16b1-inter node at a point close to the right superficial trunk (arrowhead, see A and B). In the ventral aspect of the pancreaticoduodenal region (PANC, $\mathrm{v}$ in parenteses), several arteries were dissected. 16b1-lat, No. 16b1-latero node; C, cardia (cut); FL, duodenojejunal flexure; LK, left kidney with its adipose capsule; SP, spleen. 

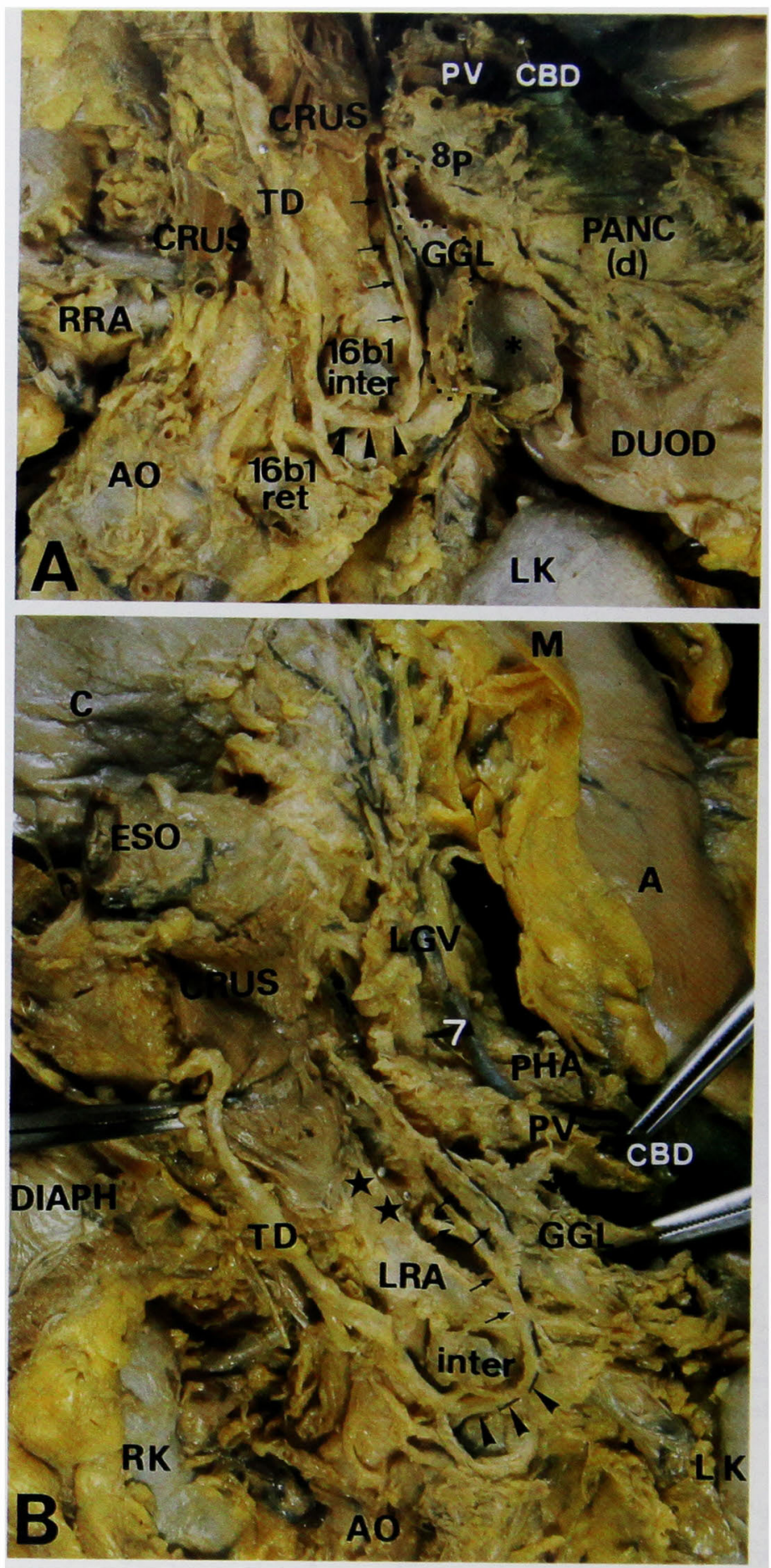

Fig. 4. Deep pathway directly merging with the thoracic duct.

A. A thick collecting lymph trunk (arrows) was found between the diaphragmatic crus (CRUS) and the celiac ganglion (GGL) in spite of poorly-developed superficial lymphatics in the dorsal aspect of the pancreaticoduodenal region (PANC, $d$ in parentheses). The deep trunk originates from No. 8p node, first runs downward but turns upward (arrowheads) near No. 16b1inter nodes (16b1 inter), sending a communicating vessel to the nodes. The trunk and other vessels from No. 16b1-retro node (16b1 ret) join to form the thoracic duct (TD). Most part of the inferior vena cava was removed except for its superior portion labeled by an asterisk. AO, aorta; CBD, common bile duct; DUOD, third portion of the duodenum; LK, left kidney; PV, portal vein; RRA, right renal artery.

A. Deeper dissection from the view in A. Arrows and arrowheads along the trunk shown in A correspond in those in B. The deep lymphatic trunk is formed from 2-3 collecting trunks originating from an area near the No. 7 node (7). No. 8p node was removed. The GGL and PV are retracted to the left side. Stars indicate the levels of the celiac (upper star) and superior mesenteric (lower) arteries. A, antrum of the stomach; C, cardia; ESO, esophagus; LGV, left gastric vein; LRL, origin of the left renal artery; M, middle portion (body) of the stomach; PHA, proper hepatic artery: RK, right kidney. 


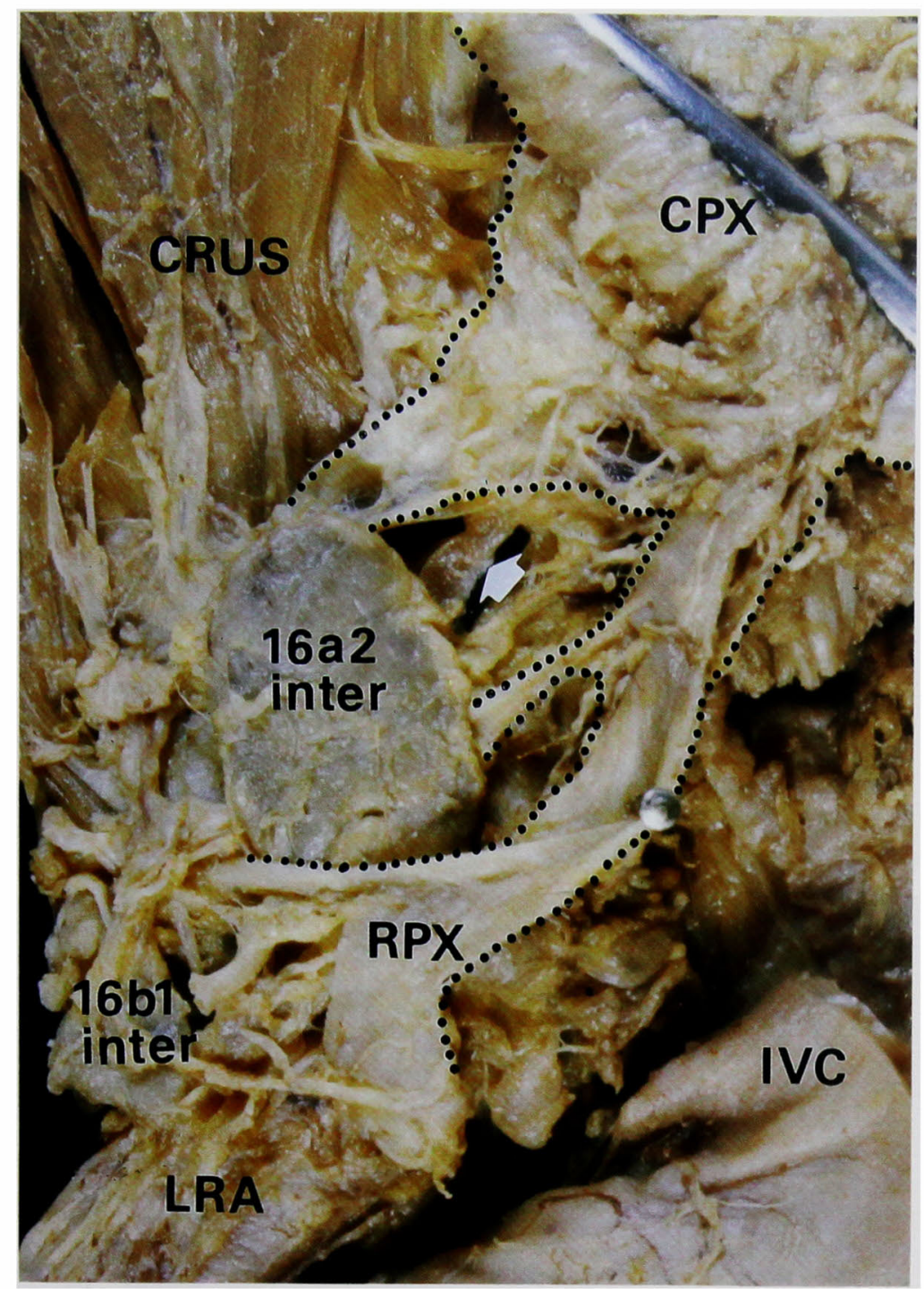

Fig. 5. Suggestive deep route between the crus and celiac plexus in a specimen without finding a long pathway.

A thick collecting vessel (white arrow) is found draining into No. 16a2-inter node (16a2-inter) after almost complete removal of the celiac plexus (CPX, enclosed by dotted lines). No. 16a2-inter communicates with No. 16b1-inter nodes (16b1 inter), but the left renal vein, interposed between those nodes, was removed. The left renal artery (LRA) and inferior vena cava (IVC) were dissected and reflected. RPX, right renal plexus.

of the limited numbers of specimens overall, the long descending pathway was dominant in frequency and rich variability in the right or dorsal side of the pancreaticoduodenal region.

\section{Discussion}

We demonstrated thick collecting vessels originating from No. 8 node and/or other pancreaticoduodenal region near Nos. $6,11,12,13,14,17$ nodes and draining directly into No. 16 nodes. We would like to stress that, in those nodes, No. 8 (or 12) was not located above (peripheral to) No. 13 but both Nos. 8 (or 12) and 13 nodes were situated in parallel as origins of the right collecting vessels. We called them the left and right long descending pathway. Moreover, corresponding to the dominance of the dorsal node groups (i.e., Nos. $8 p, 12 p$ and 13 nodes) in early metastasis of hilar cholangiocarcinoma, gall bladder carcinoma and pancreatic cancer (see Introduction), the thick collecting vessels from the dorsal nodes, i.e., the right long pathway (5 specimens), were more evident than those of the left pathway from the ventral node groups ( 2 specimens). Therefore, surgical dissection of lymphatics in the right side seems to be critically important for those primary cancers mentioned 


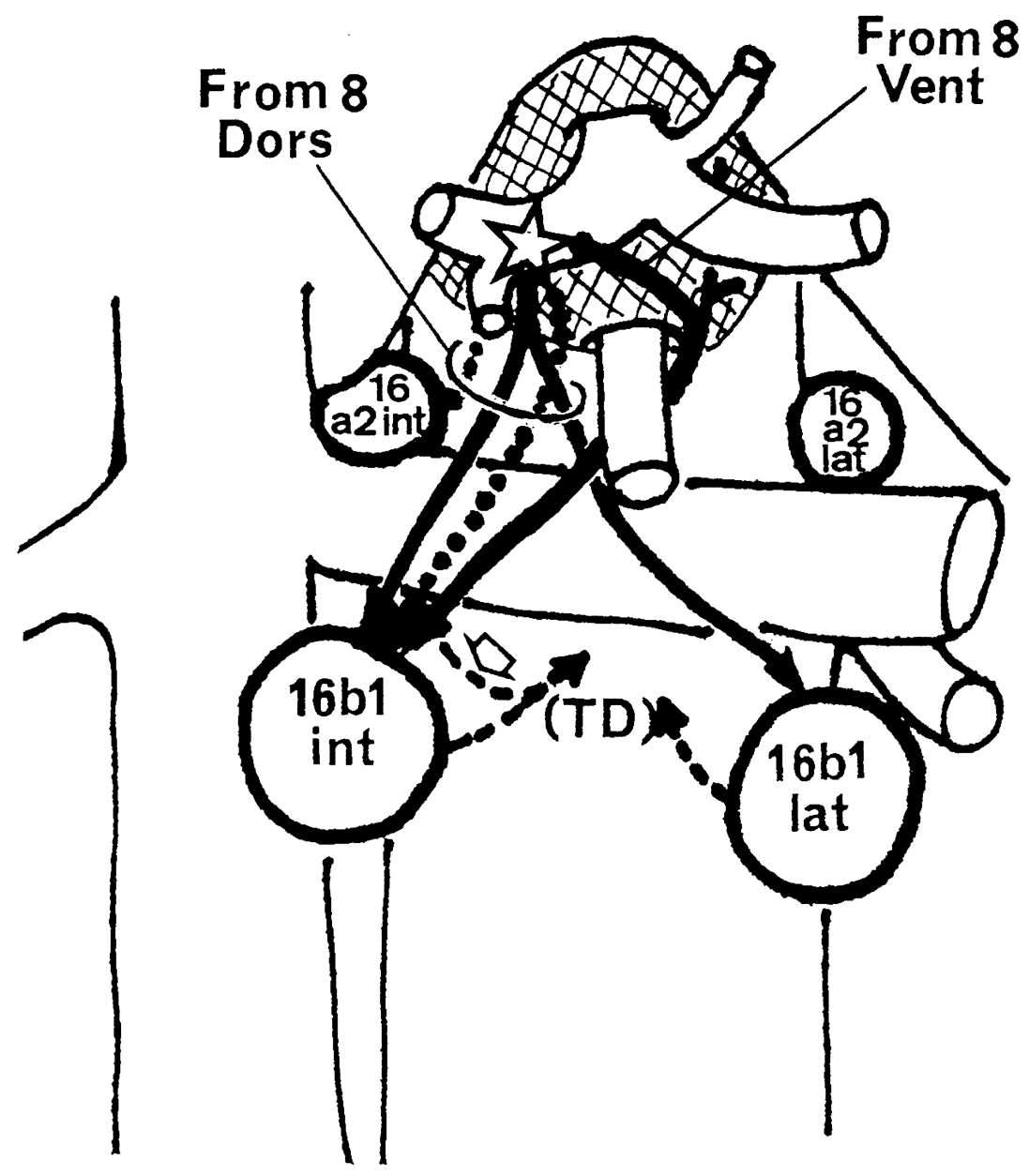

Fig. 6. Schematic representation of the long descending pathway to drain lymph from the upper abdominal viscera to the para-aortic nodes.

Viewed from the ventral side. Lymph from the upper abdominal viscera is though to be received by the upper para-aortic nodes at the level of the renal artery and vein, i.e., No. 16a2-inter, No. 16a2-latero, No. 16b1-inter and No. 16b1-latero nodes. The present study demonstrates thick collecting vessels descending to the para-aortic nodes without intercalated nodes along the course: 1) Lymphatic routes originating from the ventral/dorsal side of the pancreatic head and/or the ventral/dorsal group of nodes along the common hepatic artery (star, No. $8 \mathrm{a} / 8 \mathrm{p}$ ) in the left/right side of the celiac and superior mesenteric artery (From 8 Dors or Vent); 2) Lymphatic routes running superficial/deep to the celiac plexus and left renal vein (solid/dotted lines). One of the deep routes (wide, open arrow) directly continued to the thoracic duct (TD) without intercalation with No. 16 nodes. In addition, another deep route drained into No. 16a2-inter node in the right side. We did not find a direct route to No. 16a2-latero node.

above, although there may be a question as to whether every right pathway can be approached under Kocker's maneuver (see below, the last paragraph).

Our long pathways might correspond, mostly or partly, to the pathways proposed by Ito et al. (1991) and Ito and Mishima (1994), i.e., the cholecystoretropancreatic pathway on the right side and the cholecysto-celiac pathway on the left side. However, according to our understanding, their pathway was not based on the direct, thick and long collecting vessels but on the network composed of numerous lymphatic vessels and nodes. Therefore, surgeons can not eliminate the possibility that
No. 13 node (and even No. 14 node) is located "along" their right pathway (Kondo et al., 1998). We believe that the pathway should be a direct route to the thoracic duct origin, i.e.. No. 16 nodes, without any intercalated nodes. Actually, by using excellent injection for adult fresh cadavers, Nagai (1987) pointed out the almost same pathway as we present. However, because his dissection depended on surgical procedures, he failed to demonstrate the entire course and some missing parts remained between different fascial layers.

In the present study, the numbers of specimens examined were very limited. Therefore, another pathway, such as a left deep one, might be found in 
further dissections. Moreover, we believe that another origin of the pathway will be found in a more peripheral area than examined in the present study, such as Nos. 7 and 12 nodes. Although lymphatic valves were not evident along the course (see Materials and Methods), we believe that the origin was situated at the visceral side along the pathway and the end, near the thoracic duct (i.e., No. 16 nodes). As Sato and colleagues stated (1997), there was heterogeniety in No. 16 nodes: No. $16 \mathrm{~b} 1$ nodes (i.e., No. 16b1-inter node and No. 16b1-latero node) were the selected terminals of the pathway if the direct continuation to the thoracic duct was neglected. The diaphragmatic crus, celiac plexus and left renal vein seemed to prohibit access of the thick lymph vessel to No. $16 \mathrm{a} 2$ nodes. Even the right deep pathway seemed to be forced to go downward along the diaphragmatic crus. Sato et al. (1988b) suggested that some of the tributaries (origins) of the thoracic duct runs through the crus. However, we could not find penetration of such a thick vessel in this study.

A lymphatic drainage route running deep to the celiac plexus was also observed in the present study. Some researchers (e.g., Ito et al., 1991) previously described it but regarded it as a variaiton or an additional, less-effective route. No. 16a2-inter node was located close to the crus as well as the plexus. We speculated that the short afferents of No. 16a2-inter node, that were often observed, are a potential pathway from No. $8 p$ and/or No. $12 p$ nodes to the paraaortic region. However, the final identification of the deep node chain along the crus (i.e., No. $16 \mathrm{a} 2$ node and/or No. 9 node including Lnn. celiaco-retropancreaticae after Inoue, 1936) might require further study. Notably, the right deep route seemed to be the nearest way along the diaphragmatic crus between the left gastric arterial territory to No. 16b1-inter node. To drain the left upper area, another left pathway was reported from No. 2 node (nodes on the dorsal side of the cardia of the stomach) to the No. 16a2-latero node (Sato et al., 1988a).

The classical concept of the intestinal lymph trunk is different depending on the report, even in those from the same laboratory (Jossifow, 1906, 1930; Kutsuna, 1956; Hiraki, 1958b; Tanigawa, 1963). The difference seems to be based on the different standpoints of comparative anatomy, i.e., the question of which is the minimum common territory of the trunk in many mammals or which is the largest territory of all. According to the narrowest definition (Hiraki, 1658b), the trunk is formed by the joining of several collecting vessels from almost the entire territory of the superior mesenteric artery except for the pancreaticoduodenal region.
If translated into today's classification of lymph nodes (Japanese Research Society for Gastric Cancer, 1995), the intestinal trunk may be defined as a single efferent vessel of No. 14 nodes. However, the expanded entity (Kutsuna, 1956) includes other collecting vessels from the liver, spleen and stomach. Our left pathway might correspond to the intestinal trunk in the wider meaning. In contrast, the dominant right pathway is not consistent with any previous definition of the intestinal trunk due to its limited territory (i.e., the dorsal aspects of the pancreaticoduodenal region and hepatoduodenal ligament). Is it better to limit the intestinal trunk to a single collecting vessel? Should there only be one "trunk"? Murakami et al. (1990) defined more than 2 bronchomediastinal "trunks" in the left side and at least 3 in the right side. We considered that the intestinal lymph trunk is also able to originate from various locations and have different courses and terminations, and that there can be more than one.

According to our results, the descending pathway drained into not only the No. 16b1-inter node but also into No. 16b1-latero node. The former is accesible under Kocker's maneuver (see Introduction), whereas the latter is not impossible but diffcult to access. Moreover, complete dissection of the deep route during surgery seems to require the complete resection of the celiac plexus, in contrast to minimumly-invasive surgery (see Introduction). Although it was less dominant, another problem seems to be the left descending pathway, which is not operated on in the right-side approach. Nevertheless, the present results provided a good basis for reconsideration of the procedure, as pointed out by Kondo et al. (1998): No. 13 node is not located "along" the right superficial pathway but it is one of the origins (see also Introduction). We speculated that, during surgery for cancer at the hepatic hilum or gallbladder, a target selection as to the right, left or both pathways can be decided with detailed examinations of metastasis in hepatoduodenal ligament (i.e., which subgroups of No. 12 nodes are involved in metastasis). In other words, cancer involvement of No. 12 a node seems to demand complete treatment of the left pathway, i.e., probably standard pancreaticoduodenectomy. We are preparing another paper with respect to such practical problems based on the present results.

\section{References}

1) Abstract of the 24th Meeting of Lymph Node Metastasis Research. Jpn J Lymphology 1996; 19:36-40, 46-54. (in Japanese)

2) Deki H, Sato T. An anatomical study of the peripancreatic lymphatics. Surg Radiol Anat 1988; 10:121-135 
3) Deki $\mathrm{H}$, Sato $\mathrm{K}$, Sato $\mathrm{T}$. Lymph drainage routes from the last visceral node on the stomach to the paraaortic nodes. Shoukaki-Geka (Gastroenterol Surg) 1991; 14:153-163.

4) Durovicova J, Munka V. Lymph drainage of the stomach in man and certain mammals. Folia Morphol (Praha) 1973; 21:411-413.

5) Fujii M, Murakami G, Yamagata T, Shimada K, Hoshi H, Tanaka T. Topographical anatomy of the internal mammary lymphatics in the superior mediastinum and anterior mediastinal lymph nodes. Okajima's Folia Anat Jpn 1994; 71:99-126.

6) Henne-Bruns D, Vogel I, Lüttges J, Klöppel G, Kremer B. Ductal adenocarcinoma of the pancreas head: survival after regional versus extended lymphadenectomy. HepatoGastroenterol 1998; 45:855-866.

7) Hidden G, Hureau J. The large lymphatic vessels of the adult digestive viscera. Anat Clin 1978; 1:167-176.

8) Hiraki S. An anatomical study of the thoracic duct in human embryos. Kumamoto Igaku-Kai-Zattushi (J Kumamoto Med Soc) 1958a; 32:1008-1019. (in Japanese)

9) Hiraki S. An anatomical study of the intestinal lymph trunks in human embryos. Kumamoto Igaku-Kai-Zattushi (J Kumamoto Med Soc) 1958b; 32:987-996. (in Japanese)

10) Hiraki $S$. An anatomical study of the lumbar lymph trunks in human embryos. Kumamoto Igaku-Kai-Zattushi (J Kumamoto Med Soc) 1958c; 32:997-1007. (in Japanese)

11) Inoue Y. Über das Lymphgefässystem des Magens. Duodenums, Pankreas und des Zwerchfells. Acta Anat Nippon 1936; 9:35-117. (in Japanese with German abstract)

12) Ito $M$, Mishima $Y$, Sato $T$. An anatomical study of the lymphatic drainage of the gallbladder. Surg Radiol Anat 1991; 13:89-104.

13) Ito $M$, Mishima $Y$. Lymphatic drainage of the gallbladder. J Hepatobiliary Pancr Surg 1994; 1:302-308.

14) Japanese Research Society for Gastric Cancer. Japanese Classification of Gastric Carcinoma. First English ed, pp. 6-11, Kanehara, Tokyo, 1995.

15) Jossifow GM. Der Anfang des Ductus thoracicus und dessen Erweiterung. In Arch $\mathrm{f}$ Anat Physiol, Anat Entwickelungsgeschichte. W Waldeyer ed. pp. 68-76, Veit \& Comp, Lepzig, 1906.

16) Jossifow GM. Das Lymphgefässystem des Menschen. pp. 65-71, Gustav Fischer, Jena, 1930.

17) Kayahara $M$, Nagakawa $T$, Kobayashi $H$, Mori $K$, Nakano T, Kadoya N, Ohta T, Ueno K, Miyazaki I. Lymphatic flow in carcinoma of the head of the pancreas. Cancer 1992; 70:2061-2066.

18) Kida H. An anatomical study of the lumbar and intestinal lymph trunks and the thoracic duct in human embryos. Kumamoto Igaku-Kai-Zattushi (J Kumamoto Med Soc) 1958; 32:880-900. (in Japanese)

19) Kutsuna M. An anatomical study of the lymph trunks. Kumamoto Igaku-Kai-Zattushi (J Kumamoto Med Soc) 1956 39:1-26. (in Japanese)

20) Kondo $T$, Nimura $Y$, Hayakawa N, Kamiya J, Nagino $M$, Kanai M, Uesaka K, Yuasa N, Sano T. Reliability and limitations of lymphadenectomy of the paraaortic nodes for curative surgery of the gall bladder cancer. Jpn J Surg Soc 1998; 99:728-732. (in Japanese)

21) Mukao G. An anatomical study of the thoracic duct and its related lymph trunks in mammalian embryos including human. Kumamoto Igaku-Kai-Zattushi (J Kumamoto Med
Soc) $1954 ; 28: 769-801$. (in Japanese)

22) Murakami G, Sato $T$, Takiguchi $T$. Topographical anatomy of the bronchomediastinal lymph vessels. Arch Histol Cytol 1990; 53:219-235.

23) Murakami G, Sato I, Shimada K, Dong C. Kato Y, Imazeki $T$. Direct lymphatic drainage from the esophagus into the thoracic duct. Surg Radiol Anat 1994; 16:399-407.

24) Murakami G, Hirata K. Takamuro T, Mukaiya M. Hata F. Kitagawa S. Vascular anatomy of the pancreaticoduodenal region: a review. J Hepatobiliary Pancreat Surg 1999; 6:5568.

25) Nagai H. An anatomical and pathological study of autopsy materials on metastasis of the pancreatic cancer to the paraaortic lymph nodes. Jpn J Surg Soc 1987; 88:308-317. (in Japanese)

26) Nagai $H$, Sada $H$, Kuroda $S$. Topographical relationship between the lymphatics draining the biliary truct and the celiac nerve plexus. Tan-to-Sui (Biliary truct and Pancreas) 1991; 12:129-138. (in Japanese)

27) Nakao A, Harada A, Nonami T, Kaneko T, Murakami H, Inoue $\mathrm{S}$, Takeuchi $\mathrm{Y}$, Takagi $\mathrm{H}$. Lymph node metastasis in carcinoma of the head of the pancreas region. Br J Surg 1995; 82:399-402.

28) Pissas A. Anatomoclinical and anatomosurgical essay on the lymphatic circulation of the pancreas. Anat Clin 1984; 6:255-280.

29) Pitt HA. Curative treatment for pancreatic neoplasms: standard resection. Surg Clin North Am 1995; 75:891-904.

30) Sarrazin R, Pissas A, Dyon JF, Bouchet Y. Lymphatic drainage of the stomach. Anat Clin 1980; 2:95-110.

31) Sato K, Sato $T$. On the lymphatics passing through the hepatoduodenal ligament. Jpn J lymphology 1986; 9:69 79 . (in Japanese)

32) Sato K, Deki H, Sato T. Topographical relationship between the left cardiac nodes and the left inferior phrenic artery. Jpn J Lymphology 1988a; 10:177-190. (in Japanese)

33) Sato K, Deki H, Sato T. Topograhical relationship between the lymph nodes surrounding the abdominal aorta and renal pedicles, and the aortic hiatus. Jpn J Lymphology $1988 \mathrm{~b} ; 11: 45-55$. (in Japanese)

34) Sato T. Color Atlas of the Topographical Anatomy of Human Lymphatics. pp, 1-84, Nankodo, Tokyo, 1997. (in Japanese)

35) Tanigawa K. A study on the lymphatic system in human stomach, especially on its passage to the thoracic duct. Igaku Kenkyu (Medical Res) 1963; 33:40-64. (in Japanese)

36) Takayama Y, Ohyama S, Matudaira H, Yamaki T, Katoh K, Ohta K, Yamaguchi T, Takahashi T. Muto T. Physiological lymphatic flow originating from the stomach and colon: intraoperative experiments using carbon particles and lipid. Annals of the 4th Annual Meeting of Jpn Res Soc Clin Anat 2001; 1:10-11. (in Japanese)

37) Uesaka $K$, Yasui $K$, Morimoto $T$, Torii A, Yamamura $Y$. Kodera Y, Hirai T. Kato T, Kito T. Visualization of routes of lymphatic drainage of the gallbladder with a carhon particle suspension. J Am Coll Surg 1996; 183:345-350.

38) Yeo CJ, Cameron JL, Sohn TA, Coleman JA, Sauter PK, Hruban RH, Pitt HA, Lillemoe KD. Pancreaticoduodenectomy with or without extended retroperitoneal lymphadenectomy for periampullary adenocarcinoma. Ann Surg 1999; 229:613--624. 